\title{
Detection of Left Ventriculer Diastolic Dysfunction (LVDD) in Asymptomatic, Normotensive, ETT Negetive Newly Detected Type-2 Diabetic Patients by doppler Echocardiography
}

\author{
S.K Banerjed, P.K. Kariicakar, MA Siddique, C.M. Ahmed, A.A .Jamil, A
} .K. Sarma, M.A. Rahmam, M.A. Sultan, KMHS.S. Hauqne

\section{Background:}

Left ventricular diastolic dysfunction (LVDD) is the early manifestation of diabetic Cardiomyopathy, which is most often asymptomatic in early stage.

\section{Aim}

To detect LVDD in asymptomatic, normotensive, non-ischaemic. Uncomplicated Newly detected type-2 diabetic patients.

\section{Material and Methods}

During January 2004 through November 2003, 32 (22 men) * newly detected type-2 diabetic and 30 non-diabetic age matched subject were studied without-any evidence of complication or associated disease. All patients had negative ETT with normal global LY function. LVDD was evaluated by Doppler echocardiography by using various velocities and time intervals of mitral inflow and pulmonary venous flow, In transmitral flow, peak velocity of $E$ and $A$ waves, deceleration time (DT) of A wave, Isovolumetric relixation time (IVRT) and duration of A wave were measured. In pulmonary venous flow, peak velocity of $\S, D$ and $A R$ waves, duration of $A R$ wave was measured. The staging of LV $\mathrm{DD}$ according to severity is delayed relaxation, pseudonormalization and restrictive filling pattern.

\section{Result}

LVDD was found in 18 subjects (56\%). All of which had delayed relixation, no subjects was found to have pseudonormal or restrictive (ling abnormality, In control group, only 3 subjects $(10 \%)$ had delayed relaxation $(P<001)$. 


\section{Conclusion}

LVDD present in significant number in the entry point of type-2 diabetes mellitus who are free of clinically detectable heart disease, So, it is suggested that all patients of newly detected type-2 diabetes mellitus should be routinely screened for LVDD to detect early phase of diabetic cardiomyopathy and appropriate therapeutic intervention should be taken to arrest or reverse the process.

Bangabandhu Sheihk Mujib Medical University Dhaka, Bangladesh 